\title{
Estimating Random Errors Due to Shot Noise in Backscatter Lidar Observations
}

\author{
Zhaoyan Liu, William Hunt, Mark Vaughan, Chris Hostetler, Matthew McGill, \\ Kathleen Powell, David Winker, and Yongxiang Hu
}

\begin{abstract}
In this paper, we discuss the estimation of random errors due to shot noise in backscatter lidar observations that use either photomultiplier tube (PMT) or avalanche photodiode (APD) detectors. The statistical characteristics of photodetection are reviewed, and photon count distributions of solar background signals and laser backscatter signals are examined using airborne lidar observations at $532 \mathrm{~nm}$ using a photon-counting mode APD. Both distributions appear to be Poisson, indicating that the arrival at the photodetector of photons for these signals is a Poisson stochastic process. For Poissondistributed signals, a proportional, one-to-one relationship is known to exist between the mean of a distribution and its variance. Although the multiplied photocurrent no longer follows a strict Poisson distribution in analog-mode APD and PMT detectors, the proportionality still exists between the mean and the variance of the multiplied photocurrent. We make use of this relationship by introducing the noise scale factor (NSF), which quantifies the constant of proportionality that exists between the rootmean-square of the random noise in a measurement and the square root of the mean signal. Using the NSF to estimate random errors in lidar measurements due to shot noise provides a significant advantage over the conventional error estimation techniques, in that with the NSF uncertainties can be reliably calculated from/for a single data sample. Methods for evaluating the NSF are presented. Algorithms to compute the NSF are developed for the Cloud-Aerosol Lidar and Infrared Pathfinder Satellite Observations (CALIPSO) lidar and tested using data from the Lidar In-space Technology Experiment (LITE).
\end{abstract}

OCIS Codes: 280.3640 (lidar), 040.5160 (photodetectors), 270.5290 (photon statistics) 


\section{Introduction}

Lidar or laser radar has been used for atmospheric remote sensing since early the 1960s to measure important atmospheric parameters (wind, temperature) and constituents such as aerosols, clouds, trace gases, etc. Accurately estimating and accounting for the measurement errors (or uncertainties) introduced by various lidar system components is an important issue that must be addressed in order to ensure the reliable application of lidar data products to atmospheric studies. Well-established error-propagation theory ${ }^{1}$ is usually used in the error analysis of backscatter lidar observations. Based on this theory, an algebraic expression ${ }^{2}$ can be derived that computes the total uncertainty as a function of the various error sources. However, application of this expression requires estimates of the uncertainties attributable to each significant source.

There are two major types of uncertainty in lidar observations: random errors and bias (systematic) errors. Random errors are generally caused by random fluctuations (or noise) inherent in the measurement. For backscatter lidar measurements, these random fluctuations result primarily from: (a) quantum noise (also known as shot noise) due to the discrete nature of the incident light, charge carriers, and the interaction of light with the photodetector (i.e., photoemission); (b) thermal noise due to the random motion of electrons arising within the photodetector, load resistor and amplifier, and other noise sources (e.g., 1/f noise, etc. ${ }^{3}$ ); and (c) excess noise introduced in the multiplication process when a photomultiplier tube (PMT) or an avalanche photodiode (APD) is operated in the analog detection mode. Random errors can be reduced by averaging or by repeating the measurement. Systematic errors, on the other hand, generally arise from sources such as inaccurate calibration, nonlinearities in the photodetector response, defects in optical components, and/or a systematic electronic noise. This type of error can produce a fixed amount of bias that cannot be reduced by averaging. In contrast to the random error, however, it is sometimes possible to reduce the effects of systematic errors when their sources are known. As the focus of this paper is random error, we will not be discussing systematic errors in any further detail.

In lidar observations, the noise arising from background radiation and detector dark current, but excluding those fluctuations due to the scattering signal, is generally referred 
to as the background noise. Background noise is easily measured and is independent of range from the laser transmitter. The standard deviation of the background signal can be determined, for example, from the samples acquired before firing the laser (i.e., when there is no backscattered signal), or from the samples corresponding to very high altitudes (e.g., > $40 \mathrm{~km}$ ) where the laser backscatter is negligibly small when compared to the magnitude of the background signal. In contrast to the background noise, the magnitude of the noise associated with the scattering signal depends on the range-resolved intensity of the backscattered light, and thus needs to be estimated separately for each data sample. In this paper we will focus our discussion on this latter type of error, and on methods for estimating its magnitude.

For lidar measurements, the conventional method widely used to estimate the random error is to compute the standard deviation of a series of consecutive samples. These samples can be obtained either vertically, from sequence of consecutive range bins within a single lidar profile, or horizontally, from samples at the same range bin obtained over some number of consecutive profiles. When using these statistical techniques, however, the natural variability of the atmosphere can cause significant overestimates of the random component of the measurement error. This effect is especially severe in those areas where the atmospheric composition changes rapidly (e.g., within clouds). Given that measuring the variability of the atmosphere is one of the fundamental objectives to be realized by the use of backscatter lidar observations, it is thus highly desirable to have an error estimate that can be generated in a manner wholly independent of the ambient atmospheric content.

In this paper, we introduce the noise scale factor (NSF) to estimate the random error due to signal shot noise. The derivation of the NSF is based on the fact that when the intensity of an incident light field does not fluctuate during the time of observation (i.e., when it remains in a statistically stationary state), photons sampled during this time will follow a Poisson stochastic process. ${ }^{4,5}$ In Section 2 of the paper we review the statistical basis of photodetection. The mathematical derivation of the NSF is presented in Section 3. Practical techniques for ascertaining the correct value for the NSF are developed in Section 4. This development is illustrated via application to the lidar that will fly aboard the Cloud-Aerosol Lidar and Infrared Pathfinder Satellite Observations (CALIPSO) 
satellite $^{6}$, and tested using data acquired during the Lidar In-Space Technology (LITE) mission ${ }^{7}$. Issues of transferring the NSF from one signal domain to another, and concerns arising from averaging partially correlated samples, are discussed in Section 5. Concluding remarks and a summary are given in Section 6.

\section{Statistics of Photodetection}

\section{a. Shot Noise}

PMTs and APDs, operating either in a photon-counting mode or in an analog mode, are the standard photodetectors used for backscatter lidar observations. We will therefore focus our discussions on the statistics of photodetection using PMTs and APDs.

Even if the radiation field is of constant intensity, the number of photons arriving at the photodetector during any time increment is inherently uncertain due to the quantum nature of light. Straightforward, statistical proofs exist showing that if photon arrival rates are time-independent (i.e., they can be described as being a statistically stationary process), the total number of photons arriving during any time interval $\tau$ is Poissondistributed. ${ }^{4}$ Theoretical studies have established the correspondence between the number of photons incident on the detector and the number of photoelectrons emitted, and thus the photoelectrons also have a Poisson distribution. ${ }^{5}$ The probability of emitting $n_{\mathrm{p}}$ photoelectrons during time $\tau$ is given by

$$
p\left(n_{p}\right)=\frac{\left(\bar{n}_{p}\right)^{n_{p}}}{n_{p} !} e^{-\bar{n}_{p}} .
$$

In this expression, $\bar{n}_{p}=\tau \eta P / h v$ represents the mean number of photons emitted. $P$ is the power of the incident field, $\eta$ is the quantum efficiency of detector, $h$ represents Planck's constant, $v$ describes the frequency of the field, and $h v$ is the energy of the photon. Both here and afterwards, an overbar (e.g., $\left.\bar{n}_{p}\right)$ is used to indicate that a quantity represents a mean or average value. For a Poisson distribution, the variance is equal to the mean, so that

$$
\overline{\Delta n_{p}{ }^{2}}=\bar{n}_{p},
$$


where $\Delta n_{p}$ represents the standard deviation. The variance quantifies the uncertainty in the measurement due to shot noise. The Poisson distribution applies to light emitted from an ideal laser having deterministic intensity, or from a thermal radiation source such as the sun that has a coherence time $\tau_{\mathrm{c}}$ much smaller than the sampling time $\tau^{5}$ Examples of the photon count distributions of solar background signals and laser scattering signals are given in Figure 1Figure 1. These data were acquired by the Cloud Physics Lidar (CPL), which is an airborne, down-looking system that uses photon-counting detection and can thus provide direct photon count measurements. The data shown in Figure 1Figure 1 was obtained from daytime measurements, where detector (APD) dark counts are negligibly small when compared to the background light signal. The background signal distribution in Figure 1Figure 1(a) was compiled using 100 subsurface samples (i.e., signals containing no laser backscatter) from 1000 profiles (a total of 100,000 samples). Each CPL raw sample is acquired in a counting time period of $0.2 \mu \mathrm{s}$ (corresponding to a $30 \mathrm{~m}$ vertical resolution) and accumulated over 500 shots. This results in an effective counting time of $0.1 \mathrm{~ms}$ for each raw sample. The composite atmospheric scattering distribution (laser backscatter + background signal) shown in Figure 1Figure 1(b) was derived using six samples (range bins) from $\sim 10 \mathrm{~km}$ in the 1000 profiles (a total of 6,000 samples). For comparison, Poisson distributions having the same means as the measured data are also shown in each panel, and it is clearly seen that both the laser scattering signal and the solar background signal share the same type of distribution - Poisson.

In general, if the radiation field intensity varies with time, the photodetection statistics are governed by a compound Poisson distribution (also known as Mandel's formula), 5, 9 whose rate density is proportional to the instantaneous electromagnetic energy collected by the detector. In this case, the variance is given by

$$
\overline{\left(\Delta n_{p}\right)^{2}}=\bar{n}_{p}+(\eta / h v)^{2} \overline{\Delta W^{2}}
$$

where $W$ is the integrated optical intensity over time interval $\tau$ and $\Delta W$ is the standard deviation of $W$. The additional term in the expression for the variance, $(\eta / h v)^{2} \overline{\Delta W^{2}}$, results from the field fluctuations of the incident radiation. This term, which in 
photodetection of thermal light is sometimes called "photon-bunching noise", is a

consequence of the correlation of fluctuations in the thermal light intensity., 9 In backscatter lidar observations, this excess noise may arise from fluctuations in the laser source and/or the natural variation of the atmospheric scattering media. Fluctuations of laser output are usually small, and are monitored during the observations in order to energy-normalize the lidar data prior to subsequent analyses. As a result, the effect of laser fluctuations is ignored in our analysis. However, the variability of the atmosphere and its components, especially clouds, can be very large. As mentioned above, characterizing this atmospheric variability is one of the primary objectives of backscatter lidar measurements. We therefore do not include an atmospheric variability term in our random uncertainty estimates.

\section{b. Excess Noise (Multiplication Noise)}

For a PMT or an APD operated in the analog detection mode, the output electrons (multiplied photoelectrons) at the anode do not obey Poisson statistics, even if the incident photons (or emitted photoelectrons) do. ${ }^{10-12}$ This is because the photoelectron multiplication in these detectors is also a stochastic process, which can introduce an excess noise. In a typical PMT, the photoelectrons emitted from the photocathode are multiplied by a set of dynodes via the secondary emission of electrons. The probability distributions of the multiplication gains of these PMTs can be described by a multiple stochastic (compound) Poisson distribution ${ }^{10}$ 12. In APDs, on the other hand, photoelectrons can initiate impact ionization to produce extra hole-electron pairs, which in turn result in more hole-electron pairs as they move through the space-charge region (avalanche region or multiplying region). The photocurrent is thus multiplied. For a uniform APD having a thick multiplying region, the probability distribution of gains can be characterized analytically by a local-field theory. ${ }^{11}$

The variance of multiplied electrons can be expressed as ${ }^{10-12}$

$$
\overline{\left(\Delta n_{m}\right)^{2}}=F_{m} G_{m} \bar{n}_{m}
$$


where $n_{\mathrm{m}}$ is the number of multiplied electrons and $\bar{n}_{m}$ is the mean number of such electrons. $\bar{n}_{m}$ is determined by

$$
\bar{n}_{m}=G_{m} \bar{n}_{p}
$$

where $G_{\mathrm{m}}$ is the average gain of the multiplication and $\bar{n}_{p}$ is the mean number of photons incident on the detector. The $F_{\mathrm{m}}$ term in Eq. (4) represents the excess noise factor that is used to quantify the extra noise caused by the variability of the multiplication gain in a PMT or APD. The excess noise factor is a function of the average gain, $G_{\mathrm{m}}$, for both PMTs and APDs ${ }^{10-12}$. For PMTs, $F_{\mathrm{m}}$ normally ranges from 1 to 2 , and decreases as $G_{\mathrm{m}}$ increases. For APDs, $F_{\mathrm{m}}$ increases with increasing $G_{\mathrm{m}}$ and is normally larger than 2 . The larger excess noise introduced in the APD is due to the greater uncertainty of the APD multiplication gain. The APD gain variation arises from two sources: (1) the randomness in the locations at which ionizations may occur, and (2) the feedback process associated with the fact that both electrons and holes can produce impact ionizations as they move in opposite directions. In contrast, in standard PMTs only one carrier - electrons - causes secondary emissions (or multiplication), and this occurs only at fixed locations (dynodes). For PMTs having identical gain factor $m$ for each dynode, the excess noise factor is given by ${ }^{10,12}$

$$
F_{m}=\frac{m}{m-1} .
$$

In this case, $G_{\mathrm{m}}=m^{N}$, where $N$ is the number of dynodes. For uniformly multiplying APDs, ${ }^{11}$ the excess noise factor is

$$
F_{m}=k \cdot G_{m}+(1-k)\left(2-\frac{1}{G_{m}}\right),
$$

where $k$ is the ratio of ionization coefficients due to holes and electrons. As an example, $F_{\mathrm{m}}=1.5$ for PMTs when $m=3$, and $F_{\mathrm{m}}=5$ for APDs when $k=0.03$ and $G_{\mathrm{m}}=100$.

\section{Noise Scale Factor (NSF)}

As shown by the above discussion, there exists a proportional relation between the variance and the mean of the shot noise for both PMTs and APDs operated in either a 
photon-counting detection mode or an analog mode. Based on this proportionality, we introduce the noise scale factor to estimate the standard deviation, $\Delta x$, of the shot noise in a measurement $x$ from its mean, $\bar{x}$, using

$$
\Delta x=N S F \cdot \bar{x}^{1 / 2} .
$$

NSF has units of the square root of the units for $x$. For lidar observations using photon counting (e.g., Refs. 8 and 13), the random error due to shot noise can be estimated from the number of photon counts based on Eq. (2). In this case, $N S F=1\left(\right.$ counts $\left.^{1 / 2}\right)$ in the photon-counts domain. For the analog detection, the NSF in the multiplied-photoelectron domain is given by

$$
N S F=\left(F_{m} \cdot G_{m}\right)^{1 / 2} .
$$

For lidar observations, the data is normally sampled using a digitizer. In the digitizerreadings domain, NSF can be derived from the signal-to-noise ratio analysis for the lidar measurements (see, e.g., Ref. 14), and is computed using

$$
N S F=\left(2 e B F_{m} G_{m} G_{A}\right)^{1 / 2} .
$$

Here $e$ is the electron charge, $B \approx 1 / 2 \Delta T_{0}$ is the spectral bandwidth of the lidar receiver, and $\Delta T_{0}$ is the integration time. $G_{\mathrm{A}}$ is a gain factor that converts the anode current of the detector to digitizer counts, with the assumption that linear amplifiers are used. $G_{\mathrm{A}}$ is a product of a number of converting/scaling factors and gains.

In practice, some amount of background signal, arising from the background radiation, detector dark current, etc., is unavoidably included in the lidar measurements. Thus each digitized sample, $V$, can be written as $V=V_{\mathrm{s}}+V_{\mathrm{b}}$, where $V_{\mathrm{s}}$ represents the laser backscatter signal and $V_{\mathrm{b}}$ represents the background contribution. The overall random uncertainty for each sample is therefore the sum of the uncertainties in each of these quantities:

$$
\Delta V=\left[N S F^{2} \bar{V}_{s}+\left(\Delta V_{b}\right)^{2}\right]^{1 / 2} .
$$


In this expression $\bar{V}_{s}$ is the mean of the scattering signal, and $\Delta V_{\mathrm{b}}$ is the background noise; i.e., the standard deviation of the background signal. $\Delta V_{\mathrm{b}}$ can be measured directly from the samples where there is no laser scattering signal (e.g., subsurface samples or very high-altitude samples). Generally $\bar{V}_{s}$ is unknown. However, if the measurement is not very noisy, the uncertainty can be estimated from a single sample using

$$
\Delta V \approx\left[N S F^{2} V_{s}+\left(\Delta V_{b}\right)^{2}\right]^{1 / 2}
$$

Note that, in practice, $V_{\mathrm{s}}$ is typically derived by subtracting the measured mean value of the background signal, $\bar{V}_{b}$, from the raw digitizer reading $V$; i.e., $V_{s}=V-\bar{V}_{b}$. When computed in this manner, $V_{\mathrm{s}}$ is also a random variable, and thus an additional uncertainty, $\Delta \bar{V}_{b}$, which represents the uncertainty in the measured $\bar{V}_{b}$, must be introduced into the calculation; that is,

$$
\Delta V \approx\left[N S F^{2} V_{s}+\left(\Delta V_{b}\right)^{2}+\left(\Delta \bar{V}_{b}\right)^{2}\right]^{1 / 2}
$$

$\Delta V_{b}$ is usually determined by computing the standard deviation of a number of samples where there is no scattering signal and $\bar{V}_{b}$ is the mean of these samples. Therefore, the error in the estimate of the mean is

$$
\Delta \bar{V}_{b}=\frac{1}{\sqrt{N_{b}}} \Delta V_{b},
$$

where $N_{\mathrm{b}}$ is the number of the samples from which $\bar{V}_{b}$ is computed. This number is usually quite large, so that $\Delta \bar{V}_{b}$ is typically much smaller than $\Delta V_{b}$.

The advantages of using the NSF to estimate the uncertainties inherent in lidar backscatter measurements are illustrated by the CPL profile measurements shown in Figure 2Figure 2. To derive the conventional error estimates, standard deviations (with respect to the mean signals) have been computed for each altitude bin between $0-\mathrm{km}$ and $16-\mathrm{km}$ for a sequence of 100 consecutive profiles. These values are plotted using a dashed line. For comparison, standard deviations estimated from a single profile using the NSF technique are plotted using a solid line. The uncertainties computed using the two 
methods are generally consistent in the aerosol-free region above $\sim 1.5 \mathrm{~km}$, where only molecular scattering exists. However, in the aerosol layer between $0-\mathrm{km}$ and $1.5-\mathrm{km}$, significant overestimates appear in the uncertainties computed using the conventional method. This behavior is due to the horizontal variation of the particle concentration within the aerosol layer (i.e., the implicit inclusion of the $\overline{\Delta W^{2}}$ term in Eq. (3)). This comparison clearly shows that the conventional method can overestimate the random error. More importantly, a number of horizontally homogeneous profiles are required in order to derive accurate results using the conventional method. On the other hand, the NSF method can estimate the random error using only a single sample.

\section{NSF Measurement}

When the parameters in Eq. (10) are all known, the calculation of NSF is straightforward. $G_{\mathrm{A}}$ and $B$ can be determined accurately based on laboratory experiments, and they generally do not vary during the observation period. $G_{\mathrm{m}}$ and $F_{\mathrm{m}}$ however may vary during the observation period, in concert with changes in the lidar operating environment. An example where this situation can be expected to occur is provided by the Cloud Aerosol Lidar with Orthogonal Polarization ${ }^{6}$ (CALIOP) that will fly aboard the CALIPSO satellite. CALIOP (pronounced as "calliope") is a satellite-borne, twowavelength (532 nm and $1064 \mathrm{~nm}$ ), polarization-sensitive (at $532 \mathrm{~nm}$ ) lidar that, following its launch in early 2006, will conduct continuous observations of the atmosphere from space for three years. Two PMTs and one APD operated in analog mode are used to detect the two 532-nm polarization signals and the single 1064-nm total signal. The gains (and consequently excess noise factors) of these detectors (especially the PMTs) may change significantly during the course of the three-year mission. They may also change considerably during the launch phase from the ground to space, due to the severe vibration and huge change in temperature. Consequently, the NSF must be monitored constantly during on-orbit operations in order to make use of this factor in estimating contributions to the signals from random noise. This section discusses techniques for measuring NSF using the solar background signals, and develops operational algorithms for use by the CALIPSO lidar. Because the CALIOP detectors are operated in analog mode, and because, in general, NSF $=1\left(\operatorname{counts}^{1 / 2}\right)$ for both PMTs and 
APDs operated in the photon counting mode, we focus our discussion on the measurement of NSF for the analog detection mode of the two detector types.

As of this writing, CALIPSO has yet to be launched, hence we illustrate the algorithm development discussion using data acquired by LITE. ${ }^{7}$ LITE was the world's first spaceborne lidar, a three-wavelength backscatter system that flew aboard NASA space shuttle flight STS-64 in September of 1994. Figure 3Figure 3 presents an example of a singleshot lidar profile measured at $532 \mathrm{~nm}$ during the nighttime portion of LITE orbit 117. Like CALIPSO, LITE used a PMT for the 532-nm measurements and an APD for 1064$\mathrm{nm}$ channel $^{7}$. The LITE data system acquired 5500 range-resolved samples per profile at a $10 \mathrm{MHz}$ sampling rate (i.e., 15 meters per range bin). The background signal (DC component) was measured and recorded onboard by a background monitor, and automatically removed from each profile prior to digitization. In the figure, the return signal below $\sim 40-\mathrm{km}$ is seen to increase with decreasing height. This increase is due to the increasing atmospheric molecular number density and the greater incidence of suspended particles (aerosols). The scattering signal from the upper atmosphere ( $>\sim 40$ $\mathrm{km}$ ) is very small compared with the background signal (background radiation and dark current, etc.).

For a PMT in the analog mode, the dark noise is generally negligibly small when compared with the solar background noise during daytime measurements. For an APD in the analog mode, the dark noise (which is predominantly amplifier noise ${ }^{7}$ ) is dominant during nighttime measurements and is comparable to the solar radiation noise during daytime measurements. The NSF can then be derived, based on Eq. (8), using

$$
N S F=\frac{\Delta V_{b}}{\sqrt{\bar{V}_{b}}}
$$

when the solar radiation noise is dominant (i.e., daytime measurements), and using

$$
N S F=\frac{\left[\left(\Delta V_{b}\right)^{2}-\left(\Delta V_{d}\right)^{2}\right]^{1 / 2}}{\left(\bar{V}_{b}-\bar{V}_{d}\right)^{1 / 2}}
$$


when the dark noise cannot be ignored (nighttime measurements). In these equations, $\Delta V_{\mathrm{b}}$ and $\Delta V_{\mathrm{d}}$ represent the RMS noise of the total background signal and the component due to detector dark current, and $\bar{V}_{b}$ and $\bar{V}_{d}$ are their means, respectively. We note that Eq. (15) is an approximation of Eq. (16), valid only when the dark noise is negligibly small. In the following subsections, methods for computing each of these quantities are described. In addition, test results derived using LITE measurements at both $532 \mathrm{~nm}$ and $1064 \mathrm{~nm}$ are presented for both PMTs and APDs, and are discussed in detail.

\section{a. NSF Estimation for PMTs}

The RMS noise and the mean of the solar background signal must be derived in order to compute NSF using Eq. (15). The RMS background noise is estimated by calculating the standard deviation over a large number of samples in each profile, selected from a region where the laser scattering signal is negligibly small (i.e., above $\sim 40 \mathrm{~km}$; refer to Figure 3Figure 3). For LITE and CALIPSO, the background signal is (or, for CALIPSO, will be) derived by converting the background monitor reading from its native units into equivalent science digitizer counts. In Figure 4Figure 4(a) we present the square root of the background signal and the RMS noise derived from the high altitude region for LITE measurements at $532 \mathrm{~nm}$ (i.e., PMT detection) acquired during orbit 117. It is shown that the solar radiation background dominates the background signal for the daytime portion of the orbit. The NSF values derived using Eq. (15) are shown in Figure 4Figure 4(b). It is seen that the NSF is generally constant for the daytime portion of the orbit. However, at profile number 2200 there is a step change of $\sim 10 \%$, which is most likely due to an undocumented change in the PMT gain. The sudden spike in NSF values (to $\sim 10$ ) for the profiles from $\sim 2000$ to 2200 is due to the saturation of the background monitor digitizer, which can be seen in the flat-line segments of the square-root curve in Figure 4Figure 4(a). The NSF values calculated for the nighttime portion are generally smaller than that for the daytime portion, where the detector dark noise contributes significantly to the background noise. However, for those regions where lunar light (backscattered from dense clouds etc.) dominates the background signal, the NSFs have values similar to those computed during the daytime portion. The nighttime NSF values are also 
substantially noisier, due to the very low levels of background illumination combined with the limited resolution of the LITE background monitors.

\section{b. NSF Estimation for APDs}

Due to the presence of large amounts of dark noise, NSF measurement for an APD in analog mode is relatively complicated. The APD dark current represents the dominant noise source in the nighttime portion of the data, and is comparable to the solar background signal for the daytime portion. The computational difficulties arising from this situation are illustrated in the sequence of plots shown in Figure 5 Figure 5. The upper panel (Figure 5Figure 5(a)) shows the square root of the 1064-nm background monitor reading and the RMS noise of the background signal at $1064 \mathrm{~nm}$, computed over the same data segment shown in Figure 4Figure 4. The RMS noise is once again estimated using the samples above $\sim 40 \mathrm{~km}$, where the laser backscatter is negligible. Figure 5Figure 5(b) shows the NSF estimates that would be computed without first correcting the measurements for dark components; i.e., by using Eq. (15) rather than Eq. (16). The large NSF oscillations seen in the daytime segment of the APD data compare poorly with the consistent results obtained using the PMT measurements, and are a direct consequence of the dark noise contributions from the APD and the amplifier.

The APD NSF computed for the daytime portion using Eq. (16) with the dark components removed is presented in Figure 5Figure 5(c). The mean value, $\bar{V}_{d}$, and RMS noise, $\Delta V_{d}$, of the dark current were determined from the nighttime portion of the data, under the assumption that these quantities do not change significantly in the transition from nighttime to daytime observations. The computed NSF using Eq. (16) is generally constant. However, very large variations appear in regions where the solar background signal is quite small compared with the dark current. This is because, when such conditions occur, the magnitude of $\bar{V}_{b}$ becomes very similar to that of $\bar{V}_{d}$ in denominator of Eq. (16), and the uncertainties in their determination become bigger than the difference of their average values. These near-zero values in the denominator give rise to the very noisy behavior of the NSF estimate computed via Eq. (16) and seen on the left-hand side of Figure 5Figure 5(c). To stabilize the calculation of the NSF, a modified form of the equation is derived, such that 


$$
N S F=\frac{\Delta V_{b}}{\sqrt{\bar{V}_{b}+c}}
$$

where $c$ is a constant that satisfies

$$
c=\bar{V}_{d}\left(\frac{\Delta V_{d}^{2} / \bar{V}_{d}}{N S F^{2}}-1\right) .
$$

under the assumption that the NSF and $\Delta V_{d}^{2} / \bar{V}_{d}$ do not change for the chosen data segment. $C$ is chosen by trial so that the NSF curve is flattest over the entire data segment. The NSF determined according to Eq. (17) is also presented in Figure 5Figure 5 (c). It is seen to be constant over the entire data segment, with a mean of 1.39, and is generally consistent with the NSF computed using Eq. (16). This modified approach appears to be much less sensitive to noise when the background levels are low.

\section{NSF Application Issues}

a. Transferring NSF

The value of NSF is signal domain dependent. The formula for a linear transform of NSF from a domain $V$ to another domain $V^{\prime}=K \cdot V$ is given by

$$
N S F_{V^{\prime}}=K^{1 / 2} N S F_{V} .
$$

$K$ is a conversion factor independent of $V$ or $V$ '. The derivation of this formula is straightforward. As an example, the application of this formula to the lidar measured attenuated backscatter coefficients, which are a fundamental lidar product, is discussed below.

Raw lidar measurements are usually further processed in order to produce additional meaningful data products. The attenuated backscatter coefficients, $\beta^{\prime}(r)$, are derived by range-correcting and scaling the background-subtracted samples, $V_{s}=V-\bar{V}_{b}$, as follows:

$$
\beta^{\prime}(r)=\beta(r) \cdot T^{2}(r)=\frac{r^{2}}{C} V_{s}(r)
$$


Here $\beta(r)$ is the atmospheric backscatter coefficient (including both molecular and particulate contributions) at range $r$; $T$ is the atmospheric transmittance, which accounts for signal attenuation between the lidar and the volume of atmosphere at range $r$; and $C$ is the lidar calibration constant. $\bar{V}_{b}$ is the measured background signal, which is usually determined from the mean of the subsurface samples where there is no laser scattering signal (e.g., as for CPL and other down-looking lidars) or from the samples acquired at high altitudes (e.g., 65-80 km for the CALIPSO lidar) where the laser scattering signal due to the atmospheric molecules and particles is negligibly small. The uncertainty in $\beta^{\prime}$ due to shot noise can be estimated using

$$
\begin{aligned}
\Delta \beta^{\prime} & =\frac{r^{2}}{C}\left[\left(N S F_{V}\right)^{2} \cdot V_{s}+\left(\Delta V_{b}\right)^{2}+\left(\Delta \bar{V}_{b}\right)^{2}\right]^{1 / 2} \\
& =\left\{\frac{r^{2}}{C} \cdot\left(N S F_{V}\right)^{2} \cdot \beta^{\prime}+\left(\frac{r^{2}}{C}\right)^{2}\left[\left(\Delta V_{b}\right)^{2}+\left(\Delta \bar{V}_{b}\right)^{2}\right]\right\}^{1 / 2}
\end{aligned}
$$

or

$$
\Delta \beta^{\prime}=\left\{\left(N S F_{\beta^{\prime}}\right)^{2} \cdot \beta^{\prime}+\left(\frac{r^{2}}{C}\right)^{2}\left[\left(\Delta V_{b}\right)^{2}+\left(\Delta \bar{V}_{b}\right)^{2}\right]\right\}^{1 / 2},
$$

where $\Delta \bar{V}_{b}=\Delta V_{b} / \sqrt{N_{b}}$, and $N_{\mathrm{b}}$ is the number of the samples used to compute $\bar{V}_{b}$ and $\Delta V_{b} . N S F_{V}$ is the noise scale factor in the $V$ domain and $N S F_{\beta^{\prime}}=\left(r^{2} / C\right)^{1 / 2} \cdot N S F_{V}$ (refer to Eq. (19)) is the noise scale factor in the $\beta^{\prime}$ domain.

Note that, however, NSF is not constant in some domains. For example, in the $\beta^{\prime}(r)$ domain NSF is a function of $r$. In practice, it is usually more convenient (and less errorprone) to derive and apply the NSF in a domain in which its value is constant.

\section{b. Sample Average}

To produce high quality lidar data products, signal averaging over a number of range bins or over a number profiles (laser shots) is usually required. (Note, however, that while averaging is an effective way to reduce noise, as a trade-off it also degrades the resolution of the data.) When the samples are totally uncorrelated and $N$ samples are averaged, the 
RMS noise (standard deviation) can be reduced by a factor equal to the square root of $N$. , 10,11 Therefore, if the samples used in averaging are totally independent (uncorrelated), the random error due to noise in an averaged measurement, $V_{a v g}=\sum_{j=1}^{N_{\text {shot }}} \sum_{i=1}^{N_{\text {bin }}} V_{j, i} /\left(N_{\text {shot }} N_{b i n}\right)$, is estimated by

$$
\Delta V_{\text {avg }}=\frac{1}{\sqrt{N_{\text {shot }}}}\left\{\frac{1}{N_{b i n}}\left[N S F^{2} \cdot V_{a v g}+\left(\Delta V_{b, a v g}\right)^{2}\right]+\left(\Delta \bar{V}_{b, a v g}\right)^{2}\right\}^{1 / 2}
$$

where $N_{\text {bin }}$ and $N_{\text {shot }}$ are the number of range bins and laser shots, respectively, used to compute the average; i.e., $\quad\left(\Delta V_{b, a v g}\right)^{2}=\sum_{j=1}^{N_{\text {shot }}}\left(\Delta V_{b, j}\right)^{2} / N_{\text {shot }} \quad$ and $\left(\Delta \bar{V}_{b, a v g}\right)^{2}=\sum_{j=1}^{N_{\text {shot }}}\left(\Delta \bar{V}_{b, j}\right)^{2} / N_{\text {shot }}$.

\section{c. Correlation Correction}

Lidar design considerations (e.g., bandwidth and sampling frequency) may lead to the acquisition of samples that are partially correlated with neighboring samples. For example, the sampling interval of the LITE data is 15 meters, while the fundamental range resolution of the system is limited by the bandwidth of the lidar receiver (amplifier) to a resolution slightly greater than 30 meters (i.e., more than two sample intervals). As a result, neighboring samples (2 3 bins) in a LITE backscatter profile are partially correlated. Figure 6 Figure 6 (a) shows the autocorrelation function derived from the LITE Orbit 117 measurements. The calculation was restricted to the uppermost 2500 samples (i.e., data from above 40-km, where atmospheric backscatter is negligible), and averaged over 6000 profiles, so that the backscattered solar signal is essentially constant. The plot clearly shows that each LITE sample is at least partially correlated with the two samples before or after it.

Though the RMS noise is expected to reduce by a factor of $N^{-1 / 2}$ when $N$ independent samples are averaged, if the samples are partially correlated the correct expression for the relationship described by Eq. (23) becomes more complicated. To illustrate this, Figure

Formatted: $\mathrm{F}$ 
6Figure 6(b) presents the standard deviation as a function of number of range bins used to compute the average. All values were computed from the same data segment of the LITE orbit 117 measurements. For comparison, the standard deviation predicted by the $N^{1 / 2}$ relation is also presented. Figure 6Figure 6(b) clearly shows that the actual reduction of noise is not as large as would be predicted by the $N^{-1 / 2}$ relation. This is due to the partial correlation between the neighboring samples, as demonstrated in Figure 6Figure 6(a). The ratio of the measured standard deviation curve to the $N^{-1 / 2}$ curve is presented in Figure 6Figure 6(c) (dashed line). This ratio is larger than 1.5 when the number of samples averaged is larger than 10 .

When using correlated data, the difference between the measured and predicted values of $\Delta V_{\text {avg }}$ can be significant. Therefore, when using the NSF to estimate random error in averaged measurements, a correction is required to compensate for effects of sample-tosample correlation. Introducing the correlation correction function $f$, Eq. (23) can be modified as

$$
\Delta V_{\text {avg }}=\frac{1}{\left(N_{\text {shot }}\right)^{1 / 2}}\left\{\frac{f\left(N_{\text {bin }}\right)}{N_{\text {bin }}}\left[N S F^{2} \cdot V_{\text {avg }}+\left(\Delta V_{b, a v g}\right)^{2}\right]+\left(\Delta \bar{V}_{b, a v g}\right)^{2}\right\}^{1 / 2} .
$$

Note that signal averaging does not reduce $\Delta \bar{V}_{b, a v g}$, and that the samples acquired from different laser shots are uncorrelated, so that a correction for averaging over multiple profiles is not necessary.

The $f$ function can be either measured directly (i.e., the dashed curve in Figure 6Figure $6(c))$ or computed from the autocorrelation function using

$$
f\left(N_{b i n}\right)=\left[1+2 \sum_{m=1}^{N_{b i n}-1}\left(\frac{N_{b i n}-m}{N_{b i n}}\right) R(m)\right]^{1 / 2} .
$$

Here $R$ is the autocorrelation function, as shown in Figure 6Figure 6(a). Values of $f$ computed using Eq. (25) are also plotted (solid curve in Figure 6Figure 6(c)), and are generally consistent with the measurements (dashed curve) when small numbers of samples are averaged. Analytically derived values of $f$ are smaller than the measurements for large averages, due probably to systematic errors such as the baseline ripple and/or 
other electronic oscillations imposed in the measurement". The "photon-bunching noise" the emission rate of dark counts (a thermal emission process) in the PMT and of the backscattered solar signal intensity due to the lightly variability of the underlying atmosphere may also contribute to this discrepancy. The difference, however, is acceptably small $(<3 \%)$.

\section{Summary}

In the analysis of lidar data, there are two types of errors (uncertainties) that must be considered: random and systematic. This paper focuses on the estimation of random errors in the received signal due to noise inherent in the backscatter lidar measurement. The statistical characteristics of photodetection using both photomultipliers and avalanche photodiodes have been reviewed. In general, the distribution of sampled photons (photon counts) is a doubly stochastic (compound) Poisson distribution. The multiplication process in a PMT or an APD is a stochastic process, and hence generates excess noise. Consequently, the multiplied carriers (electrons for PMT and electron-hole pairs for APD) no longer follow the Poisson statistics even if the incident photons are Poisson distributed. For both PMT and APD, however, there still exists a proportional relation between the standard deviation (RMS noise) and square root of the mean of multiplied carriers. Based on this fact, the noise scale factor (NSF) has been introduced to estimate the random error due to the shot noise. The use of NSF greatly facilitates the random error estimation; it allows an estimate of random error for each individual sample in the lidar backscatter profile. The traditional method widely used for estimating random error computes statistics from an ensemble of lidar measurements, and its application thus requires a large number of samples. Furthermore, as shown in this work, when applying the conventional technique, an overestimation of the random error frequently results from the natural atmospheric variability. This bias error is especially acute in the measurement targets of greatest interest, such as boundary layer aerosols and clouds. 
Background noise is another important error source. This error, however, can be measured directly; it can be determined from subsurface samples where no scattering signals exist, or from samples acquired at very high altitudes (e.g., 40 to $80 \mathrm{~km}$ ) where the scattering signal is negligibly small. Two major components - background radiation signal and detector dark current - are included in the background signal. The distributions of these signals have also been investigated in this paper. The analysis using the CPL measurements at $532 \mathrm{~nm}$, which used photon-counting detection, showed that the photon counts due to the solar radiation follow the same statistics as the photon counts due to the laser scattering; i.e., Poisson statistics. Based on statistical characteristics of the Poisson distribution, algorithms have been developed for the CALIPSO lidar that use the solar radiation background signal to determine the NSF of the analog modes for PMTs and APDs. The algorithms to compute NSF from the solar background signal have been tested with the LITE data. It was shown that the NSF measurement for the PMT is largely unaffected by the dark current, because the dark current is very small when compared with the solar background signal. The NSF measurement for the APD, however, is significantly affected by the presence of dark current, because the dark current is large and may, in the presence of significant amplifier noise, behave statistically different from the optical signal. When computing the NSF for the APD, either the dark current must be subtracted from the solar signal or the modified algorithm must be used.

\section{References}

1. P. R. Bevington, and D. K. Robinson, Data Reduction and Error Analysis for the Physical Sciences, (McGraw-Hill, New York, 1992).

2. P. B. Russell, T. J. Swissler, and M. P. McCormick, "Methodology for error analysis and simulation of lidar aerosol measurements," Appl. Opt., 18, 3783-3797 (1979).

3. W. M. Leach, Jr, "Fundamentals of Low-Noise Analog Circuit Design", Proc. of the IEEE, 82, 1515-1538 (1994).

4. B. M. Oliver, "Thermal and quantum noise," Proc. IEEE, 53, 436-454 (1965). 
5. B. Saleh, Photoelectron Statistics with Application to Spectroscopy and Optical Communication, Vol.6 of Springer Series in Optical Sciences (Springer-Verlag, Berlin,1978), Chapter 5.

6. D. M. Winker, J. R. Pelon, and M. P. McCormick, "The CALIPSO mission: Spaceborne lidar for observation of aerosols and clouds," Proc. SPIE, 4893, 1-11 (2003).

7. D. M. Winker, Couch, R. H., and M. P. McCormick, “An overview of LITE: NASA's Lidar In-space Technology Experiment,” Proc. IEEE, 84, 2, 164-180 (1996).

8. M.J. McGill, D.L. Hlavka, W.D. Hart, J.D. Spinhirne, V.S. Scott, and B. Schmid, "The Cloud Physics Lidar: Instrument description and initial measurement results", Applied Optics, 41, pg. 3725-3734 (2002).

9. L. Mandel and E. Wolf, "Coherence properties of optical fields," Review of Modern Physics, 37, 231-287 (1965).

10. Z. Liu, and N. Sugimoto, "Simulation study for cloud detection with space lidars using analog detection photomultiplier tubes," Appl. Opt., 41, 1750-1759 (2002).

11. R. J. McIntyre, "Distribution of gains in uniformly multiplying avalanche photodiodes: Theory," IEEE Trans. Electron Devices, Ed-19, 703-713 (1972).

12. R. H. Kingston, Detection of Optical and Infrared Radiation,Vol. 10 of Springer Series in Optical Sciences (Springer-Verlag, Berlin, 1978).

13. Z. Liu, I. Matsui, and N. Sugimoto, "High-spectral-resolution lidar using an iodine absorption filter for atmospheric measurements," Opt. Eng., 38, 1661-1670 (1999).

14. R. M. Measures, Laser Remote Sensing, Krieger Publishing Company, Malabar, Florida, p 228 (1984). 

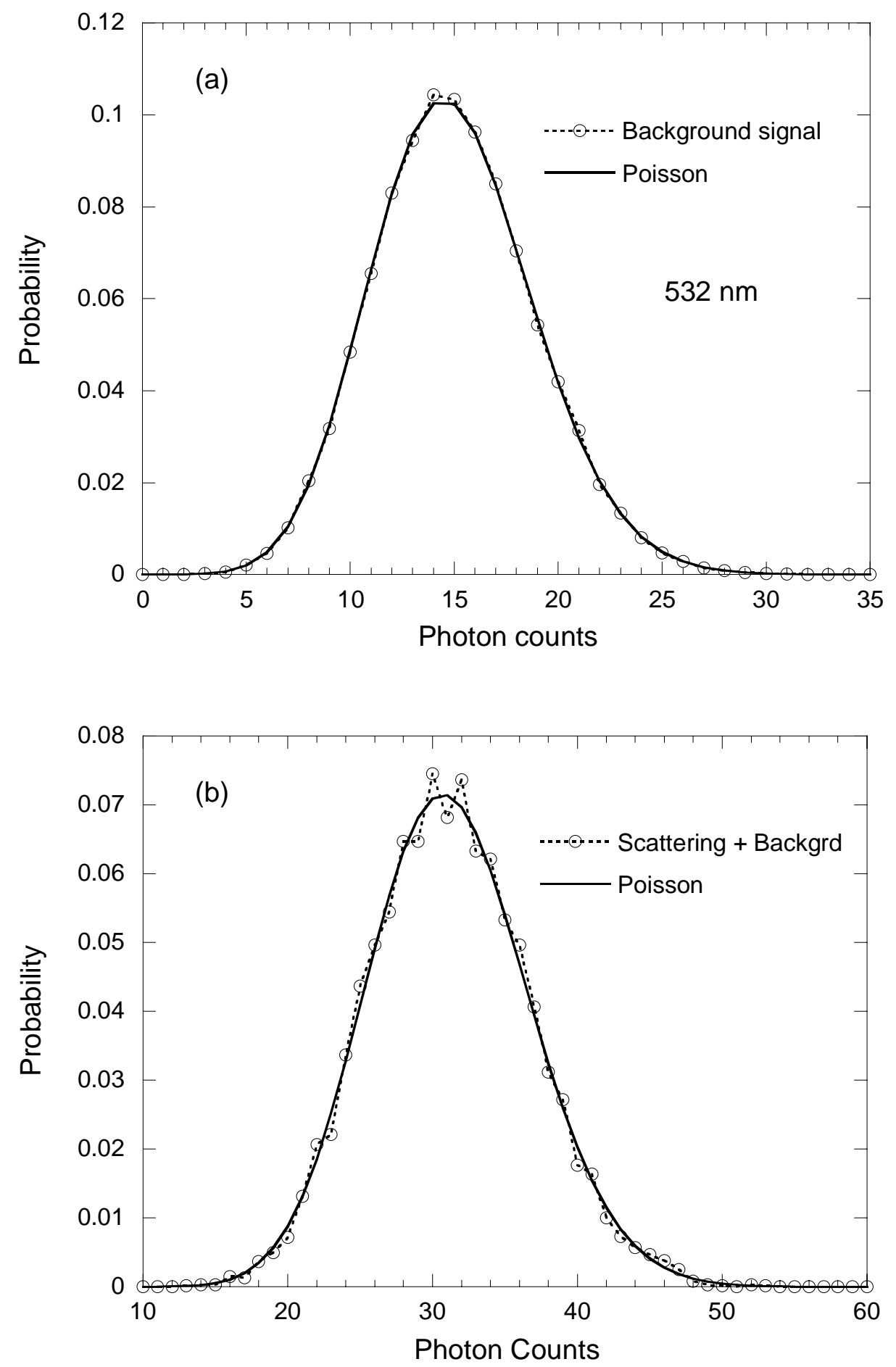

Figure 1 Examples of photon count distributions derived from CPL measurements at $532 \mathrm{~nm}$ for (a) solar background signals, and (b) laser scattering signals mixed with solar background signals. In both examples, the photon counts that comprise the input data were accumulated over an interval of $0.1 \mathrm{~ms}$. 


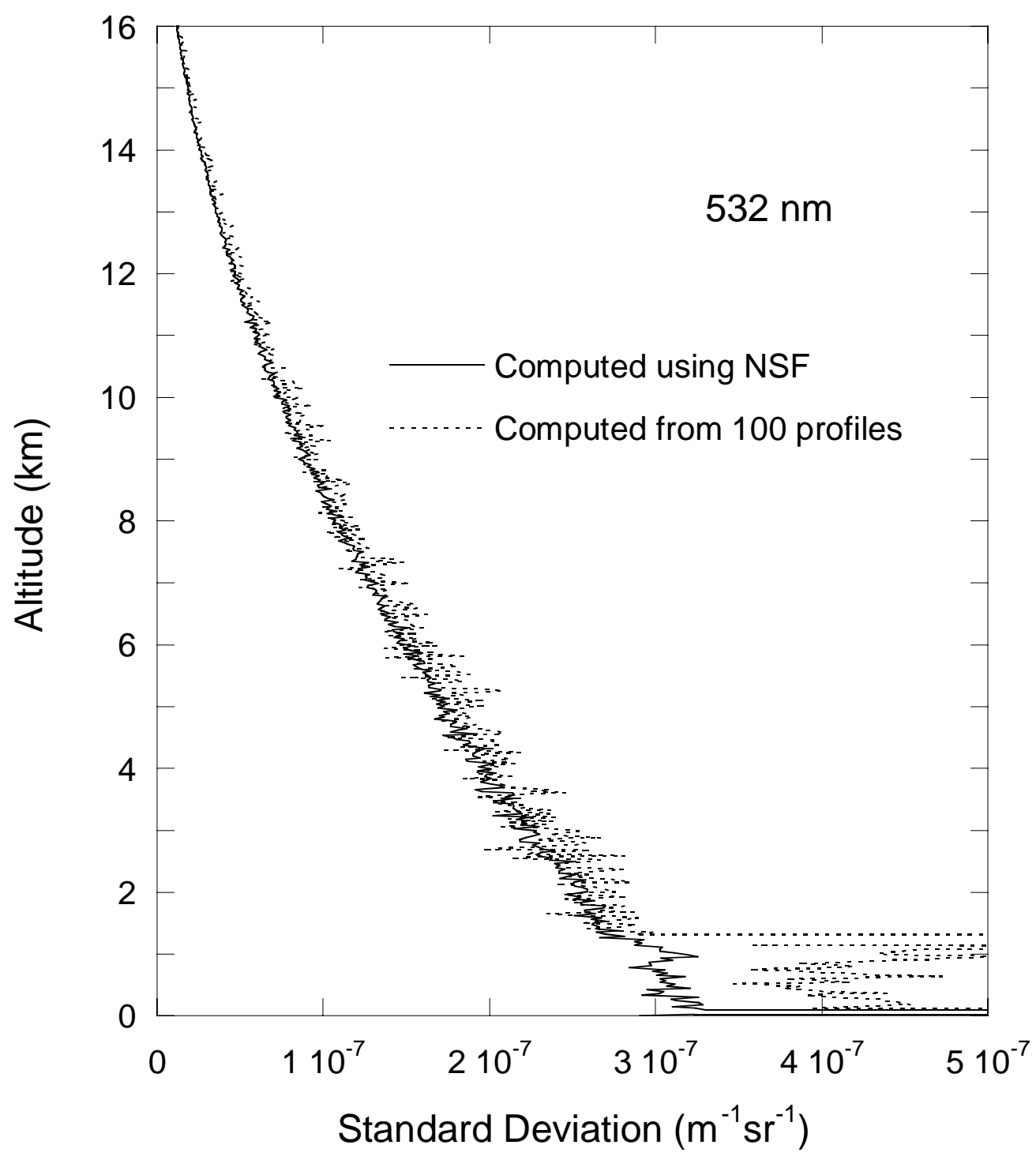

Figure 2 Examples of uncertainty estimates in attenuated backscatter $\left(\mathrm{m}^{-1} \mathrm{sr}^{-1}\right)$ derived from airborne lidar measurements using photon counting detection: standard deviations computed for each altitude bin using 100 consecutive profiles (conventional method) and using the NSF. The uncertainties computed using the conventional method are generally consistent with those derived using the NSF in the aerosol-free region (above $\sim 1.5 \mathrm{~km}$ ) where the atmospheric is relatively stable. However, due to the horizontal variability of the aerosol layer, the conventional method is seen to significantly overestimate the uncertainties below $\sim 1.5$ $\mathrm{km}$ in the profile. 


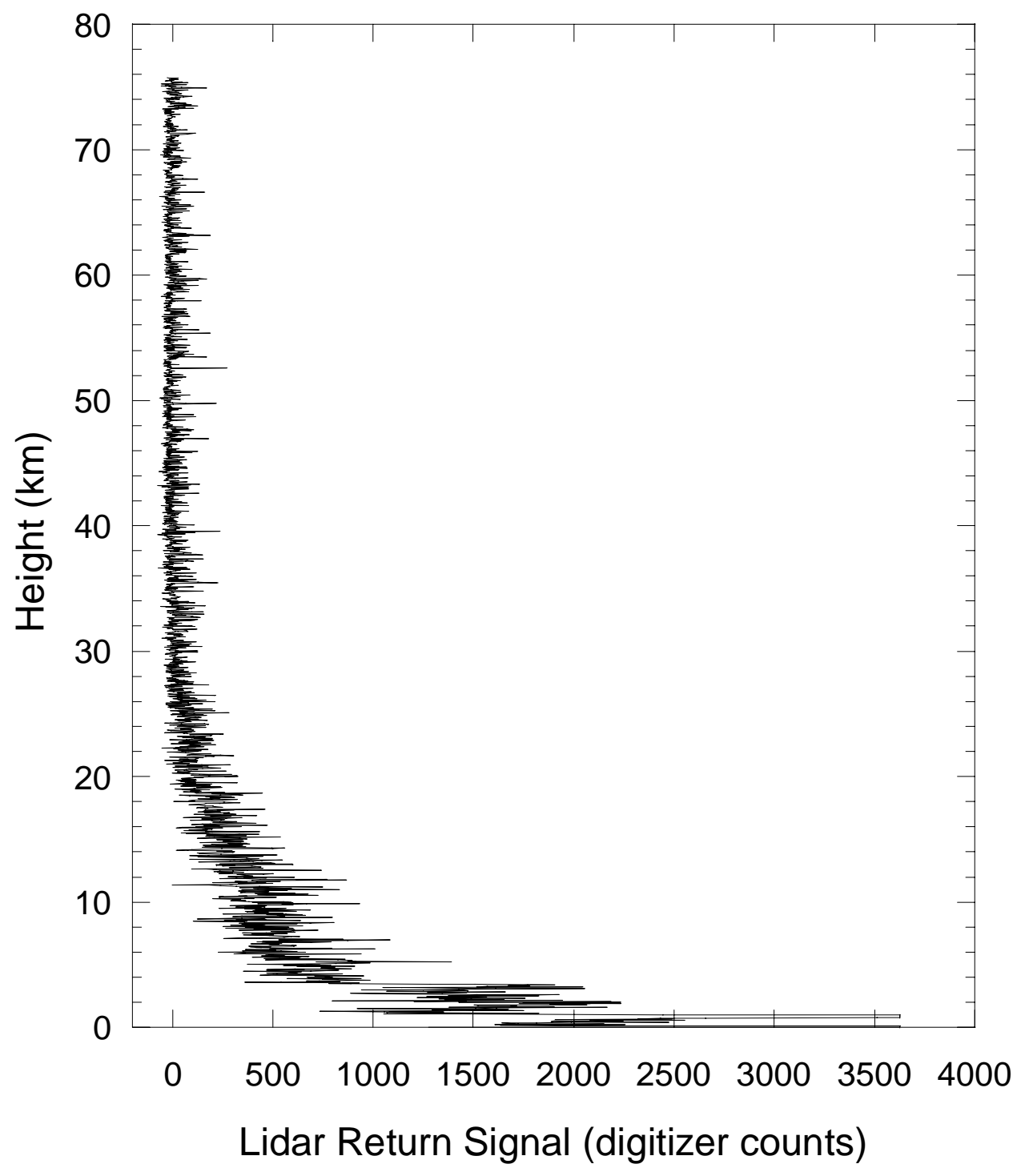

Figure 3 Single-shot lidar return profile at $532 \mathrm{~nm}$ acquired using PMT from the LITE Orbit-117 measurement. 

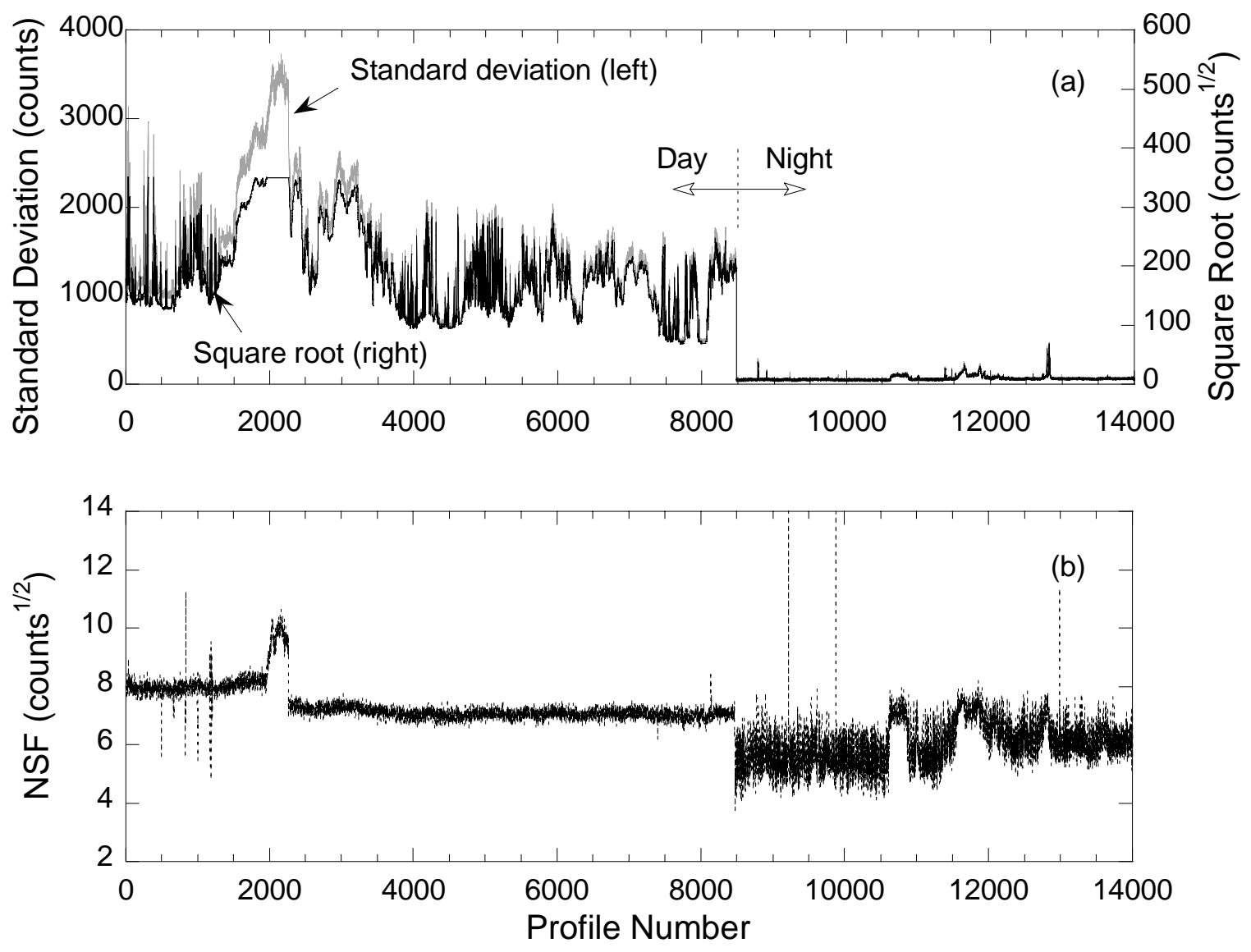

Figure 4 NSF calculations using LITE orbit 117 data acquired at 532-nm: (a) standard deviation and square root of the background signals, computed using the uppermost 2500 samples of each single-shot profile; and (b) NSF computed using Eq. (15). The arrows indicate daytime and nighttime portions of the orbit. All calculations are derived from data acquired using a photomultiplier (PMT). 

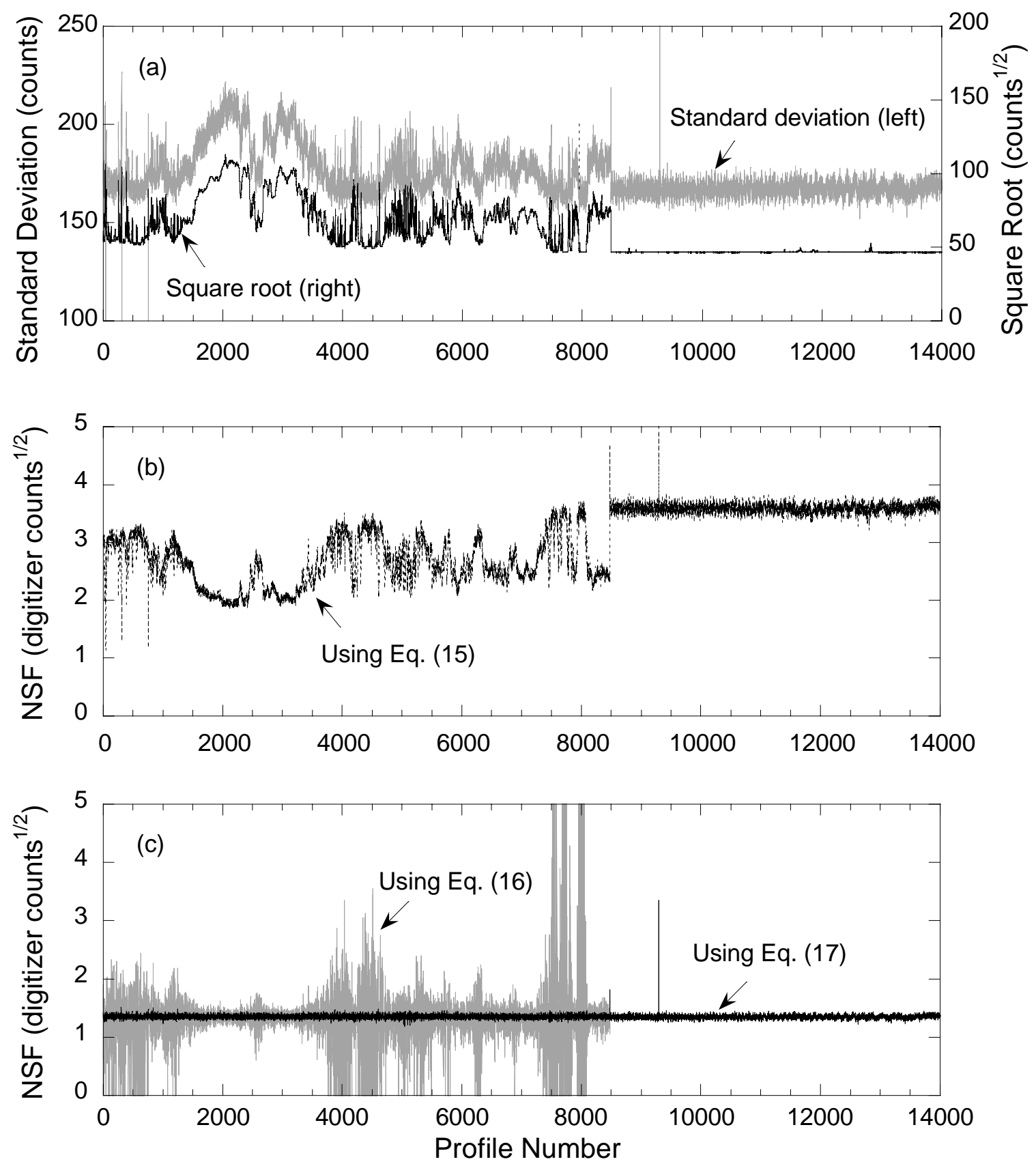

Figure 5 NSF calculations using the orbit 117 data acquired at 1064-nm: (a) the square-root and RMS noise of the background signal, computed over the same altitude regime used in Figure 4; (b) NSF computed using Eq. (15); and (c) NSF computed using Eq. 16 (pale gray line) and Eq. 17 with c =12490 (black line). All calculations are derived from data acquired using an avalanche photodiode (APD). The data segment displayed is identical to that shown in Figure 4. 

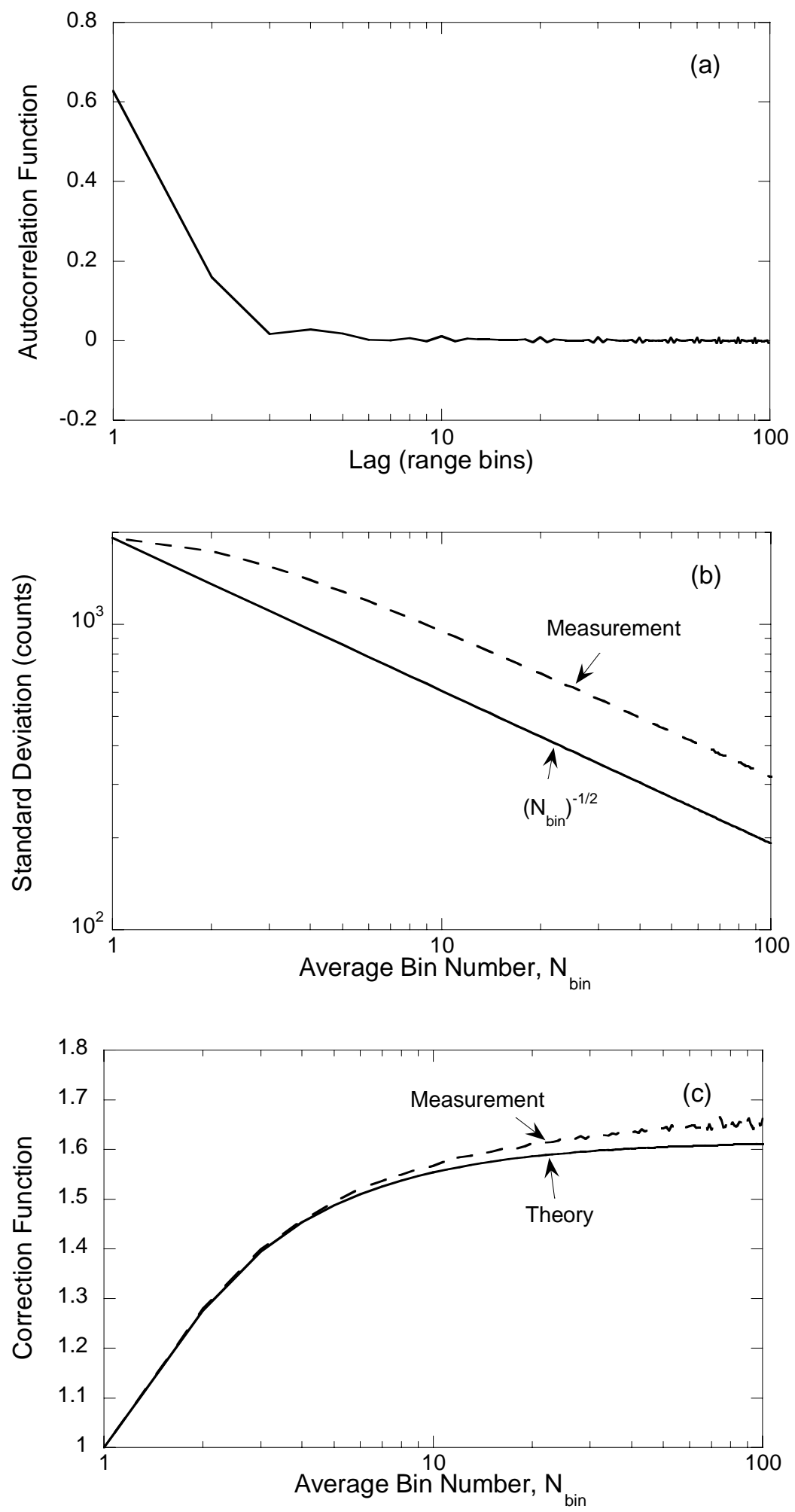

Figure 6 (a) Autocorrelation function derived from uppermost 2500 samples and averaged over 6000 profiles from the LITE orbit 117 measurement. (b) Standard deviations as a function of average bin number $N_{\text {bin }}$ from the measurement and predicted using $\left(N_{\text {bin }}\right)^{-1 / 2}$. (c) Correlation correction function. 